\title{
Optical Phase Synchronization in Coherent Optical Beamformers for Phased Array Receive Antennas
}

\author{
M. Burla, A. García García, L. Zhuang, A. Meijerink, C. G. H. Roeloffzen, \\ D. A. I. Marpaung, M. R. H. Khan, and W. van Etten \\ University of Twente, Telecommunication Engineering Group, P.O. Box 217, 7500 AE Enschede, The Netherlands. Email: m.burla@ewi.utwente.nl
}

\begin{abstract}
An optical phase synchronization system using a power feedback loop technique is experimentally demonstrated. The system allows coherent combining of signals modulated on the same optical carrier in a hybrid optical beam forming system setup.
\end{abstract}

\section{INTRODUCTION}

Integrated optical beam forming networks (OBFN) can offer several advantages compared to their electronic counterparts, such as small weight, compactness, EMI immunity, and TTD over a large instantaneous bandwidth. Among the several possible architectures, in $[1,2]$ the advantages of OBFNs using optical ring resonators (ORRs) as delay elements have been demonstrated.

Optical phase synchronization (OPS) is an important requirement in coherent optical beamforming systems. In this kind of architecture, the optical carrier produced by a common laser is split and then modulated by the RF signals from different antenna elements, delayed as desired, and combined in optical couplers. At the combining points it is fundamental that the optical signals on the two branches are in phase, so that constructive interference can occur at the detector, resulting in maximized optical power and, as a consequence, maximized RF signal power.

The issue of OPS becomes particularly sensitive when dealing with hybrid setups, where single integrated optical chips are still connected together by means of optical fibers, like in the OBFN system described in $[1,2]$, where splitting and modulation are achieved using off-the-shelf components based on optical fibers. In this case, a number of causes (such as temperature instability, mechanical stress and vibrations) occur that desynchronize the optical phases, dramatically reducing the stability of the output signal of the system.

Several solutions to this problem have been proposed and analyzed, as alternatives to the undesired manual tuning. The power feedback technique approach was chosen in this work.

Section II describes the OBFN setup, the OPS scheme and the approach for its simulation. In Section III different feedback algorithms have been analyzed and simulated in their pros and cons, and then an optimum hybrid algorithm has been chosen. Section IV describes the parameter optimization, final implementation, and testing in the OBFN experimental setup. The signal received from a television satellite has been successfully maximized at the OBFN output using the implemented technique. A conclusion is drawn in Section V.

\section{OPTICAL PHASE SYNCHRONIZATION SCHEME}

Hybrid setup A simplified schematic of the hybrid setup of the optical beam forming system considered for the optical phase synchronization problem has been thoroughly described in [3] and is represented in Fig. 1.

Martin R. Tijmes is acknowledged for the development of the OBFN simulator and for his help with the LabView software development. This work is part of MEMPHIS project, supported by Smart Mix Programme of the Dutch Ministry of Economic Affairs and the Netherlands Ministry of Education, Culture and Science.

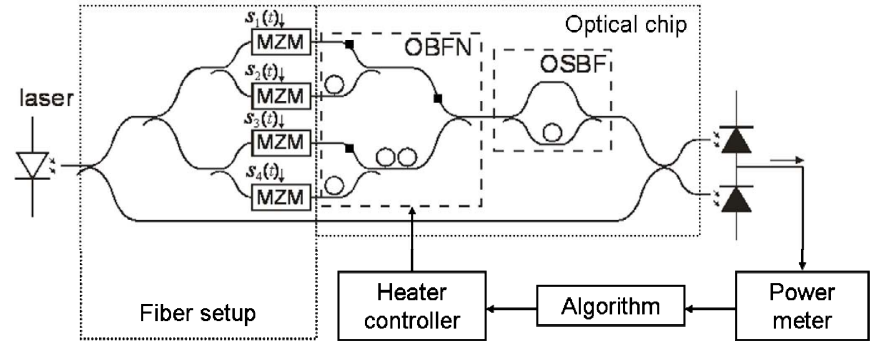

Fig. 1: Hybrid setup of the beamformer system with power feedback loop

The core of the system is an ORR-based OBFN for tunable TTD generation, an optical sideband filter (OSBF) for SSB-SC modulation, and an optical carrier reinsertion circuit for balanced coherent detection, which are integrated on a single chip $[2,3]$. The section between laser and MZMs, and between the latter and chip are all fiber-based and thus introduce the phase instability problems. The system has been designed for airborne DVB-S reception: in the following we will refer to this application.

Feedback loop design The OBFN chip has been designed to allow the compensation of phase errors in the signal paths by placing a tunable phase corrector in the upper arm before each combiner. Due to the binary tree architecture, an $N \times 1$ OBFN has $N-1$ phase correctors (Fig. 1). The input variable of the feedback loop is the RF output power, and the feedback output is then an $\mathrm{N}-1$ dimensional vector with the phase values for the correctors (actuators) as components. These are calculated on the basis of the measured output power using a feedback algorithm which will be described in the next Section.

Simulation The first step to study the feasibility of the proposed control system has been a simulation of the whole system in LabView. This software has been chosen for several reasons, among which the availability of an OBFN simulator, ease of interface with power measurement devices and with the existing OBFN control system [3]. The feedback algorithm has been implemented in MATLAB and incorporated by clever use of a script. LabView offers also visual interfaces to configure the algorithm parameters.

\section{IMPLEMENTATION OF THE FEEDBACK ALGORITHM}

The core of the feedback loop is the algorithm $A$ : it computes the new phase vector settings based on the measurement of the output power resulting from the previous phase vector:

$$
A: P\left(\bar{\varphi}_{n}\right) \longrightarrow \bar{\varphi}_{n+1}, \quad \bar{\varphi}_{n} \in[0,2 \pi]^{N-1}, P\left(\bar{\varphi}_{n}\right) \in \Re
$$

The objective of the algorithm is to calculate the optimal phase vector, defined as

$$
\bar{\varphi}_{\text {opt }}=\max _{\bar{\varphi}} P(\bar{\varphi})
$$

For this purpose, different algorithms have been analyzed. 
Hill climbing algorithm (HCA) In this iterative algorithm, after the first random initialization, the individual phase shifters are tuned sequentially, one at a time, with a maximum number of iterations each, using a simple gradient algorithm. This iterative process is suitable for a continuous tracking of the output power, but is limited by the risk of trapping in local maxima of $P$.

Genetic algorithm (GA) Genetic algorithms are implemented as a computer simulation in which a population of candidate solutions (called individuals) evolves toward better solutions [4] (Fig. 2). Each individual represents the configuration of all the $N-1$ phase shifters in the OBFN. The representation of each individual is a binary string, with $N-1$ substrings, each corresponding to the binary encoded value for a single phase shifter. In each generation, the best individuals are chosen in terms of their fitness (corresponding output power), and reproduced through bit crossover (in pairs, at a randomly chosen point) to produce the next generation. Any of the bits of the new individuals can be randomly mutated, with a probability depending on the mutation rate.

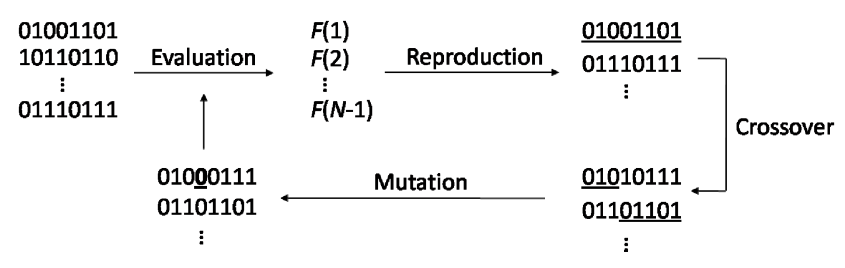

Fig. 2: Sequential steps in a genetic algorithm

Due to the evolutionary property of the GA, a minimum number of generations is required to achieve satisfactory results and as a consequence it does not provide continuous tracking. On the other hand, this algorithm has the advantage to allow a global search, that is, to find the absolute maximum of the target function, provided that a sufficient number of generations is evaluated.

Combined algorithm To limit the drawbacks and combine the advantages of the HCA and the GA, the proposed solution is a combined algorithm which starts as a GA, to avoid the local maxima, and, after a fixed number of iterations, switches to the HCA to allow tracking. After successful test at the simulator, the algorithm has been implemented in the realized system.

\section{REALIZATION AND MEASUREMENTS}

Setup The implemented setup uses a $8 \times 1$ OBFN to demonstrate the functionality of the feedback loop. For simplicity, in this work only the coherent combining of two inputs is described, even if the implemented system is able to synchronize a generic OBFN with $N$ inputs. An EM4 high-power laser is emitting $20 \mathrm{dBm}$ of optical power, which is split and fed to two MZMs, set to their $3 \mathrm{~dB}$ point. The test RF input to the modulators is generated by a network analyzer as a continuous wave at $1.545 \mathrm{GHz}$ (center of IF satellite band) with a power of $0 \mathrm{dBm}$, and then fed to a $2 \times 1 \mathrm{RF}$ splitter. The outputs of the MZMs are connected to two inputs of the OBFN, and the combiner between the two corresponding optical paths is manually set for optical and RF power equalization. The output signal from the OBFN output bypassing the OSBF is fed to a detector. The RF signal is then fed to a signal analyzer, which allows accurate power measurements on a specified band. This device is connected via GPIB to a PC which is running the LabView program implementing the algorithm described above. The computed vector of the phase shifter values is then sent via USB to the heater controller board, which commands the chromium heaters used for thermal tuning of the phase correctors. Note that in the LabView flow a delay had to be added, to account for the delays in the feedback loop (mainly due to the slow measurement speed of the analyzer) to assure that the measurement of the output power is not affected by transients. This value was set to the safe value of $500 \mathrm{~ms}$. In the future implementations the speed will be limited by the speed of the heaters (tuning time $\sim 1 \mathrm{~ms}$ ).

Experimental optimization of the algorithm parameters The test with the RF tone input was carried on in order to optimize the algorithm parameters, which are the threshold power, the fine step and the coarse step of the HCA. The insets in Fig. 3 contain the experimental tests for the optimization. The choice of a threshold power of $2 \mathrm{~dB}$ under the maximum power, a fine step of 50 units and a coarse step of 150 result in the best compromise between speed to reach the maximum and recover efficiency after desynchronization events. The controller has been built in such a way to compensate the quadratic relation between the applied voltage and phase shift, so that the number of steps is linear with the phase shift (3000 steps corresponding to $2 \pi$ ).

Synchronization of DVB-S signal A commercial satellite dish with LNB has been used to feed the OBFN with the signal received from ASTRA 19.2 E. Two IF gain stages have been used to boost the power of the $-45 \mathrm{dBm}$ signal output of the LNB. The feedback power was measured over the $36 \mathrm{MHz}$ band of a single mid-band transponder using the signal analyzer. The effect of the applied algorithm is shown by the graph of power vs time samples in Fig. 3. First, the GA runs to reach the local maximum; then, the system switches to the HCA to keep the value to the maximum also when perturbations occur.

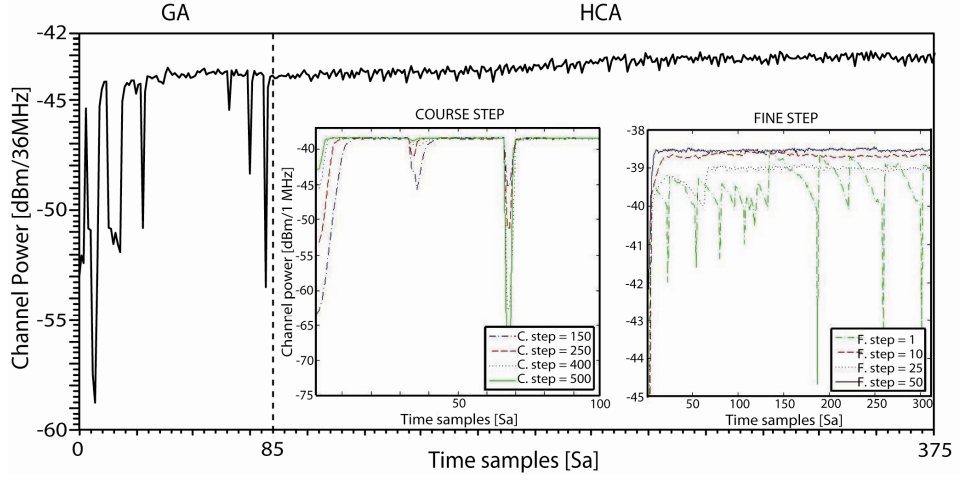

Fig. 3: Measured power output using the synchronization algorithm. The insets show the experimental optimization of the HCA course (left) and fine step (right).

\section{CONCLUSIONS}

In this paper a solution to the OPS requirement in coherent OBFNs is proposed and demonstrated. The total power feedback loop technique has been shown to be a valid method to synchronize the optical phases. Several feedback algorithms have been analyzed and simulated in their pros and cons, and then an optimum hybrid was chosen to allow, at the same time, local maxima avoidance and fast tracking against phase drifts. The performance of this solution was first simulated, optimized and finally demonstrated in the real system. This work proved that, by means of the designed feedback loop, the optical output power is successfully stabilized at the maximum value despite the drifting in the hybrid setup parameters.

\section{REFERENCES}

[1] A. Meijerink et al., Proc. $13^{\text {th }}$ IEEE/CVT Symp. Benelux (Liège, 2006).

[2] L. Zhuang et al., IEEE Photon. Tech. Lett., 15 (15), pp. 1130-1132 (2007).

[3] L. Zhuang et al., Proc. MWP 2007, paper Th. 3-3 (2007).

[4] M. Mitchell, An Introduction to Genetic Algorithms (Bradford Books, 1996). 